\title{
Les prologues des manuels scolaires pour l'enseignement du FLE comme porte-parole publicitaire des professeurs-écrivains
}

Maria Elena de la Viña Molleda

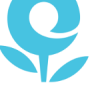

\section{OpenEdition}

Édition électronique

URL : https://journals.openedition.org/dhfles/1822

DOI : $10.4000 /$ dhfles. 1822

ISSN : 2221-4038

Éditeur

Société Internationale pour l'Histoire du Français Langue Étrangère ou Seconde

Édition imprimée

Date de publication : 1 juin 2005

ISSN : 0992-7654

\section{Référence électronique}

Maria Elena de la Viña Molleda, « Les prologues des manuels scolaires pour l'enseignement du FLE

comme porte-parole publicitaire des professeurs-écrivains », Documents pour I'histoire du français

langue étrangère ou seconde [En ligne], 33/34 | 2005, mis en ligne le 01 janvier 2012, consulté le 27 mai

2021. URL : http://journals.openedition.org/dhfles/1822 ; DOI : https://doi.org/10.4000/dhfles.1822

Ce document a été généré automatiquement le 27 mai 2021.

(c) SIHFLES 


\title{
Les prologues des manuels scolaires pour l'enseignement du FLE comme porte-parole publicitaire des professeurs-écrivains
}

\author{
Maria Elena de la Viña Molleda
}

1 Les termes prologue, préface, préambule, introduction, avant-propos, etc., sémantiquement proches, renvoient tous un discours écrit, fréquemment oeuvre de l'auteur lui-même (prologue auctorial selon Genette), qui précède l'ouvrage littéraire et le présente dans le but de retenir le lecteur ou d'attirer son attention sur certaines de ses caractéristiques. Dans le domaine des manuels pour l'enseignement du français, ces narrations préliminaires nous apportent parfois les seules informations sur différents aspects de la biographie des protagonistes et en rapport dans certains cas avec la genèse de l'œuvre. L'étude de ces pièces préliminaires, que nous considérons intéressante, sera examinée ici, de manière obligatoirement synthétique dans l'espace limité que suppose ces pages.

\section{Prologues et auto-publicité}

2 Il est manifeste que la tâche d'auteurs de manuels comporte une attitude singulièrement altruiste car elle implique l'intention non seulement de partager avec le lecteur des connaissances acquises à travers de longues années d'études mais encore d'utiliser toute sorte de procédés pédagogiques pour en faciliter l'acquisition à ce dernier. Cependant, cette activité a aussi un but commercial et dans la période où la diffusion du livre ne disposait pas des moyens publicitaires actuels, le seul procédé pour s'adresser au futur acheteur et le persuader des bénéfices que pouvait lui rapporter la lecture de son ouvrage, passait par ce message préliminaire.

3 Un des procédés utilisé à plusieurs reprisespar les auteurs de manuels - notamment jusqu'à la fin du XVIII ${ }^{\mathrm{e}}$ siècle - pour vanter la qualité de leur ouvrage consistait à mettre en avant leurs compétences en matière de langue française. Ce genre de 
publicité apparaît déjà dans la Grammatica con reglas muy prouechosas..., imprimée en 1565 et considérée jusqu'à maintenant la plus ancienne grammaire espagnole destinée à l'apprentissage du français. Baltasar de Sotomayor, son auteur, déclarait a « los muy illustres señores Corregidor y Toledo", que, pour entreprendre son ouvrage, il avait estimé suffisantes les notions de français qu'il avait tirées de l'usage et de la conversation en français ${ }^{1}$. Le sévillan Antonio del Corro (Seville 1527-Londres 1591), dont la vie est connue surtout à cause de son activité calviniste, inaugure les grammaires bilingues avec la publication à Oxford, en 1586, d'un traité de Reglas Gramaticales para aprender las lenguas española y francesa, confiriendo la una con la otra... Dans la dédicace (1586: V) adressée à l'illustre patricien génois Horatio Palavicino, il signale qu'il apprit le français lorsqu'il enseignait sa langue maternelle au roi de Henri de Navarre. Un siècle plus tard, Pedro Pablo Billet, sans doute conscient du fait que sa nationalité apparaîtrait comme une garantie pour le lecteur déclare, dans la préface de sa Gramática (1687), son origine parisienne. L'introducteur de la Gramática de la lengua francesa dispuesta al Uso del Real Seminario de Nobles, le jésuite Juan de Campo Verde, affirme en $1728^{2}$ que Núñez de Prado, son auteur, a passé sept ans de sa vie à la cour de Paris et il signale que la perfection de son langage faisait l'admiration des Français euxmêmes. La même année, apparaît l'œuvre posthume de Francisco de la Torre y Ocón intitulée Nuevo methodo breve, util y necessario para aprender a escribir entender $y$ pronunciar las dos principales lenguas, Española y Francesa, où il mentionne un long séjour à Lyon. Ce séjour vraisemblablement aurait été à l'origine de la culture française qu'il possédait $(1728: 13)$.

\section{Prologues et sources}

4 En plus de mettre en évidence leur bagage linguistique, souvent ces auteurs transmettent également, dans leurs pages préliminaires, des informations sur les sources consultées préalablement à la rédaction de leur manuel. C'est ainsi que A. del Corro (Seville 1527-Londres 1591) révèle que les règles qui y figurent ne sont pas originales : il les a recueillies trente ans auparavant lorsqu'il enseignait (1586: V). Un demi-siècle plus tard, Diego de Cisneros nous surprend quelque peu quand il assure que sa Grammatica francesa en Español (1635)est la seule qui existe pour l'apprentissage du français alors qu'il y en aurait, selon lui, plusieurs en français ${ }^{3}$. L'auteur feindrait-il pour vanter sa propre œuvre ou ignorait-il effectivement l'existence de ses devanciers? Cette tradition continue avec F. de la Torre y Ocón, traducteur de langues de la Suprême Inquisition, qui avoue avoir examiné les grammaires de RegnierDesmarais, du père Buffier ainsi que tous les traités consacrées à la didactique de la langue espagnole (1728:12). En revanche, J. Nuñez de Prado ne précise pas de titres d'ouvrages. Pourtant, dans son discours destiné « au lecteur ", il déclare s'être nourri des publications existantes pour postérieurement se servir de «de lo bueno que he hallado en quantas han llegado à mis manos porque en materia de lenguage, no se permite à un particular inventar mas» (1760: Al lector [s.n.]). Des termes quasi identiques ${ }^{4}$ sont utilisés en 1801 par J. A. Gonzalez Cañaveras dans la Vraie méthode pour apprendre les langues françoise et espagnole ce qui peut laisser supposer qu'il a lu Nuñez. Une année après, dans le Magazin des commerçants pour l'étude des langues espagnole et françoise, ce même pédagogue, qui se dit «né à Madrid, des Sociétés de Vizcaye et de Seville, Directeur d'une Académie de Langues et Sciences, que le feu Roi Charles III ordonna établir à Cadix, pour l'éducation de la jeunesse : ensuite Trésorier des Maisons 
Royales de San Ildefonso, Balsain, et Riofrio » (1802: 25), reconnait ne jamais être sorti d'Espagne et avoir conçu son manuel avec la seule «l'application à l'étude ». P. N. Chantreau (1741-1808), on le sait, auteur d'un important manuel pour l'enseignement $\mathrm{du}$ français réédité à maintes reprises pendant le $\mathrm{XIX}^{\mathrm{e}}$ siècle, critique dans son Arte de hablar bien el francés (1781) tous ceux qui ont écrit des grammaires françaises en espagnol, hormis Nuñez de Prado (1728) ou Galmace (1748). Cependant, à propos d'aspects qui représentaient des difficultés, il a ressenti la nécessité de recourir à Wailly, à Dumarsais, à Valart, à Fromant, etc ${ }^{5}$. De son côté, M. Bouynot, (1815) avertit qu'il s'est écarté de certaines grammaires et même de celle qui est considérée comme «la plus complète " pour suivre des auteurs illustres comme Wailly, Restaut, Vanier, Dumarsais, l'Homond, Duclos, Girard, Mr. L'Abbé D'Olivet (1815 : VII).

\section{Prologue et élargissement du public usager des manuels au XVIII et début du XIX ${ }^{\mathrm{e}}$ siècle}

5 Si jusqu'à de la seconde moitié du XVIII ${ }^{e}$ siècle le français était essentiellement l'apanage de la cour et de l'aristocratie ainsi que de quelques hommes d'affaires, l'influence de l'Illustration développera en Espagne l'intérêt pour cette langue, captivant une élite intellectuelle enthousiaste qui cherchera dans les publications du pays voisin les racines de la modernité. Don Josef Cayetano de Luaces y Somoza, dans l'approbation des Rudimentos de la Lengua Francesa de Pablo Francisco Rousseau, témoigne déjà en 1754 que « son tantos los Curiosos è inteligentes de el Idioma Francés, que puede decirse forman ya en el centro casi de España una Colonia Francesa » (1754: [s.n.]). De même, Chantreau déclarera, quelques années plus tard, que "la lengua francesa, hoy tan universal en las Cortes, es parte de la buena educación de la juventud, del estudio de los literatos, y de la curiosidad de las demás gentes; siendo muy util para cualquier carrera que se emprenda, y adorno y gala en los que no se dedicaren a ninguna. » (1797-III)

6 Cette "démocratisation" du français émeut L. Besses (s.a.) qui se préoccupe alors d'assigner à son livre un prix compétitif ${ }^{6}$. Il se produit dans ce contexte une demande croissante de leçons, comme le signale A. González Palencia ${ }^{7}$. Cette dernière entraînera un développement de l'industrie des manuels. Pendant tout le $\mathrm{XIX}^{\mathrm{e}}$ siècle le français conservera sa prééminence en Espagne, dépassant les autres langues étrangères. D’après J. Mendizábal (1846), ce phénomène serait dû à la richesse de ses ouvrages originaux et à celle de ses traductions.

El idioma francés es el que más se cultiva entre nosotros: nada mas puesto en razón siendo Francia una nación vecina, y su lengua un repertorio universal de gran riqueza en originales y traducciones. (1846: VII).

7 Ce sentiment, partagé par plusieurs "ilustrados », le sera aussi par d'autres auteurs de manuels comme Bordas (1842). ${ }^{8}$ Cependant, dans la corporation elle-même, Antonio de Capmany (Barcelone 1742-Cadix 1813) s'éleva contre de semblables discours. Cet auteur défendit avec véhémence, dans son Nuevo Diccionario Francés Español (1805), la suprématie de la langue espagnole, s'opposant de la sorte à ceux qu'il considère des ignorants, incapables d'admettre cette évidence :

Los mas desafectos á nuestra lengua, hallan, según dicen, mas exâcta y copiosa la francesa para las materias filosóficas y científicas, en cuya traducción tocan la esterilidad de la castellana. Estos españoles bastardos confunden en primer lugar la 
esterilidad de su cabeza con la de su lengua, sentenciando que no hay tal ó tal voz, porque no la hallan. ¿Y cómo la han de hallar, si no la buscan, ni la saben buscar? Y dónde la han de buscar si no leen nuestros libros? Y cómo los han de leer, si los desprecian? Y no teniendo hecho caudal de su inagotable tesoro, cómo han de tener á mano las voces que necesitan? (1805:XIV-XV) d'obsession. Ce manque de sympathie pour le français est spécialement frappant chez un homme qui consacra une partie de sa carrière à l'élaboration d'ouvrages destinés à la traduction de l'espagnol en français'. Ses excès atteignirent un tel degré que son œuvre Respuesta del organista de Móstoles a la carta de su amigo el sacristán de Berrinches (1786) ne reçut pas l'approbation du Conseil de la Censure: qui allégua qu'il n'était pas (Serrano Sanz, 1906: XV, 45-46) :

tolerable en buena política, ni en buena crianza, que se vilipendie a los escritores de otra nación, y mucho menos que se insulte con palabras de mofa y menosprecio al nombre y cuerpo de la misma nación. Así, es preciso borrar de este papel, para que pueda correr con licencia y decencia, las expresiones de burla, de provocación y aun de sedición que he notado contra Francia.

Si les jugements négatifs sur la France et sa langue furent courants à toutes époques dans notre pays, le commencement de la guerre d'Indépendance (1808) supposera la division de la population en deux factions fortement opposées. D'un côté, se trouvaient les dénommés « afrancesados » qui collaborèrent avec Joseph I, dans l'espoir de pouvoir mettre en oeuvre la politique des lumières s'appuyant sur la Constitution française de 1791, mais le peuple se méfie de ces derniers, les considérant comme des antipatriotes et des traîtres. De l'autre côté, se situaient les partisans de la monarchie qui luttaient pour se libérer du joug français. Parmi ces derniers, on distinguait d'abord les « rénovateurs» ou «jovellanistas» -anciens admirateurs de la culture françaiseplus modérés et qui, tout en soutenant les héritiers de la dynastie des Bourbons, souhaitent le rétablissement de la monarchie et ce, dans le cadre d'une réforme constitutionnelle qui en devait en finir finisse avec trois siècles de despotisme royal, ensuite les « absolutistes » qui exigeaient le retour de l'Ancien Régime sans concessions et seront les principaux acteurs de la propagande antifrançaise. Nul doute que dans ce climat hostile la publication d'un manuel pour l'apprentissage du français deviendra un défi. Pourtant ni situation politique troublée ni le manque de stabilité de l'enseignement de cette matière scolaire dans les différents programmes n'interrompent le développement de l'industrie des manuels de cette langue. Les professeurs, dont la situation économique sera souvent difficile, trouveront dans la publication de leurs propres ouvrages un moyen d'élargir leurs revenus. Dans cette aventure littéraire, le succès commercial sera inégal, certains ne dépassant pas la première édition tandis que d'autres multiplièrent les tirages comme Galmace, Tramarría, Cornellás, Bergnes, etc. ou bien sûr, Pedro Nicolas Chantreau (déjà mentionné) dont l'Arte sera en outre, remanié, complété ou corrigé par de nombreux auteurs comme J. Alarcón (1845), L. de Alemany (1826), A. Bergnes de las Casas (1845), L. Bordas (1842), J. D. Campos (1926), L. Cot (1825), A. Dieguez (1889), P. Dupuy (1836), A. Galbán (1863), G. Hamonière (1824), M. M. Nuñez de Taboada (1824), P. Puiggari (1841), P. M. de Torrecilla (1850) ou T. Vert y Mercader (1853) au cours du XIX siècle. 


\section{Prologue et didactique au $\mathrm{XIX}^{\mathrm{e}}$ siècle}

10 À partir du XIXe siècle les prologues vont jouer un rôle de propagande bien plus important qu'avantpour attirer d'éventuels acheteurs. Si pendant les siècles précédents l'expression des compétences francophones de ces auteurs suffisait pour assurer le mérite de l'œuvre, dorénavant cette qualité devra être renforcée par l'expérience. La professionnalité des auteurs et leurs efforts pour perfectionner les procédés didactiques seront constamment mis en avant dans la majorité des prologues. Nous aurons ainsi des informations intéressantes sur la formation des auteurs et leur pratique comme enseignants mais aussi sur divers aspects relatifs à la genèse de leurs manuels. Certains auteurs prétendront avoir découvert la méthode miracle pour enseigner la langue avec une rapidité et une facilité remarquables. Le plus surprenant de ce point de vue, V. Alcober y Largo (Alcañiz 1822-?) se déclare dans sa Método lexiológico y hermenéutico para aprender lengua francesa (1857) épouvanté par la perte du temps consacré à l'étude du français. Il assure posséder « un método especial » qui lui a permis d'apprendre, pendant les trente premières années de sa vie, quarante langues ou dialectes. Pour l'attester il inclut (1857:5) dans l'introduction un certificat de succès à des examens d'hébreu, arabe, allemand, anglais, latin, italien, français et portugais devant plusieurs supérieurs et employés du secrétariat d'interprétations de langues. Il fait aussi part de ses connaissances en grec, chaldaïque, samaritain, rabbinique, hollandais, suédois, danois, flamand et autres langues ou dialectes sans pouvoir en apporter la preuve faute d'examinateurs. F. Vila e Iglesias professeur de La Asociación defensora del trabajo nacional y de la clase obrera avec une expérience issue de son long séjour en France, écrit sa Gramática de la lengua francesa (1849) avec pour objectif de " ahorrar tiempo y molestia á todos los que por gusto ó por necesidad se dediquen á este estudio» (1849: I). Il garantit qu'en suivant ses règles on pourrait arriver au bout de trois mois à prononcer parfaitement le français ainsi qu'à le parler et à l'écrire avec exactitude. R.E.L. Delaborde (1863), qui n'était pas initialement professeur de français mais médecin, propose un système qui fixe à 80 le nombre de jours nécessaires pour l'apprentissage de la langue française, plusieurs «individus" de tout âge et toute condition ainsi que les internes du Collège d'aveugles ayant bénéficié de son application pendant les 19 ans où il s'est consacré au français (1863 : VI-VII). Face à l'optimisme de ces enseignants, nous rencontrons d'autres qui préfèrent ne pas générer de faux espoirs, comme Z. Sauzeau (1845)qui affirme que « el conocimiento de una lengua no se logra sino a fuerza de comparación y de largos estudios»(1845: V) ou encore M. Bouynot (1815)qui s'adressant à l'élève, lui explique que «con todo, si á ti, querido discípulo te parece mucho, y muy difícil de aprender lo que contiene, has de saber que á mí me ha costado muchísimo mas trabajo el ponerlo todo como lo encuentras » $(1815: V)$. Cet auteur ne sera pas le seul a constater l'effort nécessaire à l'élaboration d'une œuvre de ce genre puisque Vila é Iglesias avertit qu'il n'a pas hésité à se lancer dans ce "difficile et pénible» travail, encouragé par l'absence en Espagne d'une grammaire française complète et bien faite. $(1849: 1)$

11 En général ce dessein sera l'objectif principal de tout auteur. A. Ascaso y Pérez en 1863, J. García Ayala en 1883 ou J. M. Roques en 1896 prétendent avec leurs ouvrages avoir comblé un vide observé durant leurs années consacrés à l'enseignement. J. García Ayuso de sa part considérait que jusqu'à la publication de son Traductor de Francés 
(1879) aucun manuel ne réunissait les conditions qui devaient enrichir une œuvre de ce genre (1879 : VII).

Entre los diversos libros de trozos franceses que han visto la luz en España, no encontramos uno que reuna las condiciones que deben adornar á una obra de esta clase.

12 Mendizábal apprécie certains grammairiens comme F. de Tramarría, R. J. Dominguez et F. Anglada, quoiqu'en même temps il soit conscient du maigre profit obtenu des cours de français en Espagne. À son avis il faudrait chercher la responsabilité de cet échec dans le choix de la méthode, revendiquant l'objectif communicatif (avant la lettre) comme axe principal de l'enseignement, au détriment de l'étude de la grammaire. Son manuel empruntera alors les principes de M. Robertson: « una experiencia de veinte y cinco años ha convencido á la Europa ilustrada». (1846 : VII). La méthodologie à « objectif pratique », d'après la terminologie utilisée par Ch. Puren (1988), se consolidera tout au long du XIXe siècle faisant perdre à la grammaire sa prééminence. C. Soler y Arqués déclarera en 1889 «Sabemos que la gramática sola no enseña á hablar ni á escribir [...]. La gramática es, acaso, la lima que pule; pero no puede ser nunca la fábrica que nos dé el primitivo producto que, andando el tiempo ha de pulirse» (1889: 3-4). Tous ces commentaires, suggérant d'une manière respectueuse le manque d'efficacité des manuels élaborés par leurs prédécesseurs, deviendront un procédé très répandu, visant à refouler la concurrence, généralement avec élégance, et à se faire une place sur le marché. Mais il y aura toujours une minorité qui osera émettre ses critiques sans ambages. Le plus attaqué Chantreau, dont la grammaire sera considérée comme «embrollada et indigesta " par Torrecilla (1853: VII) ou lourde et pourvue de règles grammaticales sans rapport entre elles, par Alemany (1826: III).

\section{Conclusion}

13 Nous avons pu constater que la presque totalité des ouvrages consultés étaient précédés d'un texte préliminaire, généralement autographe, ce qui traduit l'impérieuse nécessité des professeurs-auteurs de s'adresser à leur lecteur. En fait, faute d'autres procédés publicitaires, les prologues ont constitué le seul moyen de persuader le futur acheteur des qualités des manuels. Des nos jours, l'étude de ces écrits fournit des renseignements aussi bien sur la biographie de ces auteurs que sur leur démarche professionnelle. Nous avons noté que le principal argument utilisé à toutes les époques a été le discours sur le moi professionnel rétrospectif. Jusqu'au dernier tiers du XVIIIe siècle la divulgation des compétences linguistiques, des stages en France ou des sources grammaticales consultées pour l'élaboration des manuels suffisaient à garantir la qualité de l'ouvrage. Les bouleversements économiques et sociaux qui se sont produits à la veille du $\mathrm{XIX}^{\mathrm{e}}$ ont touché aussi l'enseignement des langues modernes. L'institutionnalisation de l'éducation et l'élargissement d'un public désireux d'acquérir des connaissances pratiques ont entraîné une rénovation méthodologique. Ces professeurs, pour qui le marché de manuels est devenu lucratif, ont changé de stratégie pour capter l'attention des lecteurs et ils ont insisté dans leurs prologues, sur l'originalité de la méthode utilisée, l'expérience professionnelle et le succès obtenu par rapport à la concurrence, manifestant ainsi le processus grandissant de professionnalisation. 


\section{Bibliographie}

\section{Sources primaires}

ALARCóN, J. (1845), Novísimo Chantreau ó Completa gramática francesa : dividida en tres partes, revisada, corregida y aumentada por Juan Alarcón, Madrid, J. Viana Razola.

ALCOBER Y LARGO, V. (1857), Método lexiológico y hermenéutico para aprender lengua francesa, Madrid, Imprenta de M. Minuesa.

ALEMANY, L. DE(1844), Colección de AA. Franceses compuesta para la mas cabal instrucción de la juventud, Madrid, Librería de Doña Antonia de Sojo.

------(1826), Gramática francesa titulada el nuevo Chantreau: reformada, corregida, aumentada y arreglada a los progresos del idioma en las lecciones de Francisco Tramaría, escrita y publicada por Lorenzo de Alemany, Madrid, imprenta de Aguado.

ANGLADA, F. (1844), Gramática de la lengua francesa para uso de los españoles, Barcelona, Joaquín Verdaguer.

ASCASO Y PÉREZ, A. (1863), Método Progresivo de Traducción francesa, ó sea lecciones escogidas de literatura y de Moral, en prosa y en verso, sacadas de los Mejores hablistas franceses, y acomodados a cualquier gramática, Guadalajara, Imprenta de D. Elías Ruiz y Sobrinos.

BERGNES DE LAS CASAS, A. (1845), Novísimo Chantreau ó Gramática francesa en la que se han enmendado cuantas ediciones del Chantreau se han hecho hasta el día... Barcelona, Juan Oliveres.

BESSES, L. (s.a.) Veinte lecciones de francés, Madrid, Juan Muñoz Sánchez, editor.

BILLET, P.P. (1687), Gramática francesa dividida en dos partes, Amberes, Henrico Cornelio Verdussen. BORDAS, L. (1842), Compendio de la Gramática Francesa de P. N. Chantreau o elementos de la gramática francesa dispuestos para uso de la juventud, Barcelona, imprenta de Don Manuel Saurí.

BOUYNOT, M. (1815), Lecciones prácticas de Lengua Francesa o Nuevo Método para enseñar y aprender con más facilidad el idioma Francés, Valencia, Manuel Muñoz y Compañía.

CAMPOS, J. D. (1926), Gramática Francesa. Adaptada para los colegios hispano-americanos, por Pedro Nicolas Chantreau. Nueva edicion corregida y aumentada por... Paris, Garnier hermanos.

CAPMANY A. DE (1776), Arte de traducir el idioma francés al castellano, Madrid, Don Antonio de Sancha.

-----(1805), Nuevo diccionario Francés-Español, Madrid, imprenta de Sancha.

CHANTREAU, P. N. (1781), Arte de hablar bien Francés o Gramática completa dividida en tres partes.... Madrid, Antonio de Sancha.

CISNEROS, D. [Fray Diego de la Encarnación] (1635), Grammatica francesa en Español, Madrid, Emprenta del Reyno.

CORRO, A. DEL (1586), Reglas gramaticales para aprender la lengua española y francesa, confiriendo la una con la otra según el orden de las partes de la oración latinas, Oxford, Joseph Barnes 
COT, L. (1825), Rudimentos gramaticales en francés y en español extractos de la gramática de Chantreau para aprender la lengua francesa, Barcelona,Viuda de Pla.

DELABORDE, R.E.L. (1863), Nuevo Método teórico y práctico de lengua francesa, Madrid, Imprenta del autor.

DIEGUEZ, A. (1889), El Nuevo Chantreau ó Gramática hispano-francesa : método teórico-práctico en el cual se halla refundido, ampliado ... con una clave de los temas...por Andrés Diéguez Alonso, Barcelona, Librería de Bastinos; Madrid : Fernando Fe.

DOMÍNGUEZ, R. J. (1844), Nueva gramática francesa compuesta para el uso de los españoles y consultada con los mejores autores, Madrid, Imprenta T. Aguado y Cía.

DUPUY, P. (1836), Arte de hablar bien francés, ó Gramática completa..., con un suplemento por Mr. Dupuy, Barcelona, imprenta y librería de Francisco Oliva.

GALBÁN, A. (1863), Arte de hablar bien francés, ó Gramática francesa para uso de los Espanoles..., con un suplemento..., por Don Pedro Nicolas Chantreau. Nueva edicion revista y corregida por A. Galbán, Paris, Garnier hermanos.

GALICIA AYALA, J. (1883), Gramática Francesa y Método teórico-práctico arreglado en sesenta lecciones para que puedan aprender á leer, escribir y hablar el francés aún los que carezcan de nociones gramaticales, Valladolid, Imprenta, Librería Nacional y Extranjera de los Hijos de Rodríguez, Libreros de la Universidad y del Instituto.

GALMACE, A. (1748), Llave nueva y universal para aprender con brevedad y perfección la lengua Francesa, Madrid, en la oficina de G.Ramírez [édition consultée: 1800, Madrid, Imprenta de D. Plácido Barco López].

GARCÍA AYUSO J. (1879), El traductor francés ó colección de obras escogidas de la Literatura Francesa, en prosa y en verso, ordenadas y anotadas, Madrid, Administración; Paris, Maisonneuve et $C^{\text {ie }}$.

GONZÁLEZ CAÑAVERAS, A. (1801), Almacén de principiantes para el estudio de las lenguas española y francesa, Tomo II, Madrid, En la oficina de Mateo Repullés.

HAMONIÈRE, G. (1824), Arte de hablar bien francés, ó Gramática completa... por Don Pedro Nicolas Chantreau. Nueva edicion revista y corregida por G. Hamonière, Paris, Imp. de J. Smith.

MENDIZÁBAL, J. (1846), El Robertson español ó sea curso práctico teórico de lengua francesa, Madrid, Imprenta de D. Antonio Yenes.

NUÑEZ DE PRADO, J. (1728), Gramática de la lengua francesa dispuesta al Uso del Real Seminario de Nobles, Madrid, Alonso Balbás [édition consultée: 1760, Madrid, Imprenta de la viuda de Manuel Fdez y del Supremo Consejo de la Inquisición]

NUÑEZ DE TABOADA, M. M. (1824), Arte de hablar bien francés, ó gramática completa, dividida en tres partes...con un suplemento...por Don Pedro Nicolas Chantreau. Nueva edición revista y corregida por M. Nuñez de Taboada, Paris, Seguin.

PUIGGARI, P. (1841), El Novísimo Chantreau, ó Arte de hablar bien francés, gramática compuesta por D. Pedro Nicolas Chantreau, y ahora... emendada y en gran parte refundida por Pedro Puiggarí, Perpignan, J.-B. Alcine

ROQUES, J. M. (1896), Novísimo Curso Completo de Francés para todos los discípulos de lengua española. Práctica y teoría. Facilidad y rapidez, Madrid, Imprenta de José Perales y Martínez.

ROUSSEAU, P. F. (1754), Rudimentos de la Lengua Francesa o Extractos de preceptos breves, y claros para su prompta inteligencia, Valladolid, Imprenta de Alonso Riego. 
SAUZEAU, Z. (1845), Nueva gramática de la lengua francesa, Madrid, Imprenta de Miguel de Burgos.

SOLER Y ARQUÉS, C. (1889), Lecciones de lengua francesa, Madrid, Librería de la viuda de Hernando yC..

SOTOMAYOR, B. DE (1565), Grammatica con reglas muy prouechosas, y necessarias para aprender a leer, y escriuir la lengua Francesa, conferida con la Castellana. Con un estilo de escribir, hablar y pronunciar las dos lenguas, el Frances en Castellano, y el Castellano en France, Barcelona, Antonio Lacaualleria

TORRE OCÓN, F. DE LA (1728), Nuevo methodo breve, util y necessario para aprender a escribir, entender y pronunciar las dos principales lenguas Española, Francesa. Madrid : imprenta de Juan de Aritza

TORRECILLA, P. M.(1850), Chantreau Reformado, Paris, librería de Rosa, Bouret y C ${ }^{\mathrm{a}}$.

TRAMARRIA Y CARRANZA, F. DE (1829), Gramática francesa para uso de los españoles, Madrid, imprenta de Moreno.

VERT Y MERCADER, T. (1853), Compendio de la gramática francesa para uso de los españoles, sacado de la de D. Pedro Nicolás Chantreau, por T. Vert y Mercader, Igualada, Imprenta Jover y Serra.

VILA E IGLESIAS F. (1849), Gramática de la lengua francesa para uso de los españole, Barcelona, Imprenta y librería de la viuda é hijos de Sierra.

\section{Sources secondaires}

FERNÁNDEZ FRAILE, Mํ-E. et SUSO LOPEZ, J. (1999), La enseñanza del francés en España (1767-1936), Granada, Método.

GENETTE, G. (1987), Seuils, Paris, éditions Seuil.

GONZÁLEZ PALENCIA, A. (1948), Eruditos y Libreros del siglo XVIII, Madrid, sucs. De J. Sánchez Ocaña y Cía.

PUREN, C. (1988), Histoire des méthodologies de l'enseignement des langues, Paris,Clé international.

SERRANO SANZ, M. (1906), «El Consejo de Castilla y la censura de libros en el siglo XVIII », Revista de archivos, bibliotecas y museos, XV.

\section{NOTES}

1. Dans l'édition de 1624 il se présente comme "carme déchaussé » du couvent de Douai. Cf. Lépinette 1998 y 2000.

2. Nous avons consulté l'édition de 1760 qui inclut aussi l'Approbation de Juan de Campo Verde.

3. «Si bien se hallan muchas Gramáticas en Francés, de pocos años a esta parte; para aprender español, sola esta, impressa ya otra vez en Douay, Vniversidad del Condado de Flandes, año MDCXXIV ay en español para aprender francés con método doctrinal breue y claro ». (1635: [s.n.]).

4. «Dexar de confesar [...] que se ha valido de lo bueno que ha encontrado en ellas, porque en materia de lenguaje no se [...] ningun particular inventar mas ». (1801: X)

5. «Me opongo enteramente en muchas partes de esta obra, á los que han escrito gramáticas francesas en español; y me veo precisado a guiarme por Wailly, Dumarsais, Valart, Fromant, etc., en los asuntos en que cabe duda ú omisión; y en otras me refiero literalmente á Nuñez ó Galmace, cuando las reglas que dan son claras y seguras ». (1797: V-VI)

6. «La aceptación que en todas las clases de la sociedad tiene el francés, hace que sean muchos los que por necesidad tengan que sacrificarse para la adquisición de gramáticas de gran coste en 
su mayoría; á ese fin, no solo he procurado ser parco en el coste de estos apuntes, sino que para evitar un nuevo gasto de libro de traducción, acompaño trozos de buenos escritores franceses, muy convenientes para la lectura, traducción y análisis ». ([s.a.] : 8)

7. Dans Eruditos y Libreros del siglo XVIII, González Palencia signale les noms des professeurs de français qui sollicitent un permis municipal pour ouvrir une école privée.

8. El esmero con que los franceses se han dedicado á cultivar todo género de conocimientos tan útiles como necesarios, ha dado á su habla la prerrogativa que goza entre las demás lenguas vivas.

En efecto, no hay literato, no hay comerciante que no se halle iniciado en la traducción del francés, ó mejor dirémos; todo el que desea figurar en la sociedad se ve precisado á dedicarse al estudio del idioma francés como base de todos los demás. (p. V.)

9. Il publia le Dictionnaire déjà mentionné et Arte de traducir el idioma francés al castellano...(1776)

\section{RÉSUMÉS}

C'est surtout à partir des années soixante que l'on commence à éprouver un certain intérêt pour connaître la manière d'agir dans les écoles pendant les siècles précédents. Pourtant la biographie des principaux acteurs du fait didactique reste souvent inédite. Les prologues des manuels scolaires conservent parfois les seuls témoignages directs des professeurs qui exerçaient aussi comme auteurs et dans lesquels ces derniers manifestent leurs inquiétudes, leurs besoins, leurs revendications, leur statut dans la société, etc. Mais aussi, ils se servent de leurs prologues comme réclame publicitaire pour attirer l'attention sur leurs textes et en vanter leurs qualités par rapport à ceux de leurs concurrents. Notre travail consistera à analyser la nature des discours de ce point de vue publicitaire.

It has been primarily since the 1960s that we have seen interest in understanding the way schools were managed and run in previous centuries. Nevertheless, the experiences of the key educational participants of the period remain largely untold. The forewords of educational manuals often express the only written experiences of teachers; their concerns, their needs, their demands, their thoughts on their status in society, etc. But these forewords were used primarily as marketing matter for the manuals, to exalt the virtues of their publication against those produced by competitors. Our work will consist of analyzing the nature of of these arguments used effectively to promote the sale of their works.

\section{INDEX}

Keywords : French teacher, institutionnalisation, disciplinarisation, linguistic and paedagogical competences, professional profile, manuals, Spain, XIXth century

Mots-clés : professeur, compétences linguistiques et didactiques, institutionnalisation, profil professionnel, formation, disciplinarisation, manuels, Espagne, XIXe siècle 
AUTEUR

MARIA ELENA DE LA VIÑA MOLLEDA

Universidad de Granada 